\title{
Intestinal Obstruction Caused by Retained Surgical Swab
}

\author{
Urooj Akmal*, Abdul Qaiyoume Amini and Shahida Perveen Afridi \\ Department of Surgery, Dow University of Health Sciences, Karachi, Pakistan.
}

\begin{abstract}
Retained surgical swab (RSS) is an infrequent and avoidable complication after any type of abdomino-pelvic surgeries which leads to increased morbidity of patient as well as the cost of treatment. We report one such case of a 32 years old female patient who presented with signs and symptoms of acute intestinal obstruction, 1 year after a cesarean section. On exploration a mass was found in the region of terminal ileum which was resected followed by end to end anastomosis. Upon dissection of the mass, a large surgical swab was found inside the lumen of the gut. The mass which is formed around a retained surgical swab is known as Gossypiboma. Complete intra-luminal migration is very rare and a delayed complication of RSS and is difficult to diagnose due to vague presentation and inconclusive imaging. Surgical intervention is mainstay of treatment although it may be removed endoscopically in accessible parts of the gut and may even pass with defecation without any intervention.
\end{abstract}

Keywords: Gossypiboma, retained swab, intestinal obstruction. doi.org/10.21089/njhs.11.0034

\section{INTRODUCTION}

Retained surgical swab (RSS) is an infrequent and avoidable complication after any type of abdomino-pelvic surgeries which leads to increased morbidity of patient as well as the cost of treatment. It may present in early post-operative period with surgical wound infection, wound dehiscence or abscess formation and may present months to years after surgery with a mass $[1,2]$ Intestinal obstruction is a very rare and late consequence of RSS when the swab migrates completely into the lumen of the intestine and stuck in a narrow portion [3, 4]. Here we report a rare case of intestinal obstruction caused by surgical swab left in the abdominal cavity after a Cesarean Section one year back.

\section{CASE REPORT}

A 32 years old female patient was presented to emergency room with vomiting, central abdominal pain and distention for 3 days along with absolute constipation. Patient had undergone a Cesarean Section 1 year back and since then she was having complains of abdominal pain, distention and vomiting specially after heavy meals. On examination, she was a young lady of average height but thin built, looking distressed with signs of dehydration. There was a firm to hard mass about $12 \times 10 \mathrm{~cm}$ in peri-umbilical region with mild tenderness. Initial lab workup showed hemoglobin $(\mathrm{Hb})$ of $102 \mathrm{gm} / \mathrm{dL}$ and slightly raise white cell count $(10400 / \mathrm{mm} 3)$. Abdominal radiographs showed dilated small bowel loops with air fluid levels.

*Address correspondence to this author at the Department of Surgery, Dow University of Health Sciences, Karachi, Pakistan.

E-mail: uroojakmal@gmail.com
A working diagnosis of acute on chronic intestinal obstruction was made and patient was shifted to emergency operation theatre. On exploratory laparotomy, a mass (about $8 \times 10 \mathrm{~cm})$ was found in the region of the terminal ileum $(2$ feet proximal to ileocecal junction) wrapped with omentum and adhered to surround gut loops. Adhesiolysis was done and the mass was resected followed by end to end anastomosis of healthy ileal loops. Upon dissection of the surgical specimen, a large surgical swab was found inside the lumen of the ileum. (Fig. 1).

Rest of the bowel loops and other abdominal viscera did not show any abnormality so the abdomen was closed after thorough washout with normal saline. Patient had a smooth recovery and discharged on 7 th post op day.

\section{DISCUSSION}

A mass which is formed around a retained surgical swab (RSS) is also known as Gossipyboma [1]. Despite much emphasis on surgical swab counts and use of radio-opaque markers in surgical swabs followed by post-operative targeted $\mathrm{x}$-rays of operative site, the cases of gossipyboma are still being reported from every part of the world. Many such cases are also hidden and remain unreported due to fear of medico legal consequences and adverse publicity [2].

RSS initiates a foreign body reaction within the abdominal cavity. This foreign body reaction may be of two types. First is exudative which is more severe and presents early with sepsis and wound dehiscence due to abscess or cyst formation. The other is a slower and less severe fibrous reaction which remains asymptomatic for months and years 
or cause only mild non-specific signs and symptoms [1]. It may lead to adhesions, encapsulations, ulcers, fistula formation and transmural migration into the lumen of the gut causing acute or subacute intestinal obstruction [2, 3].
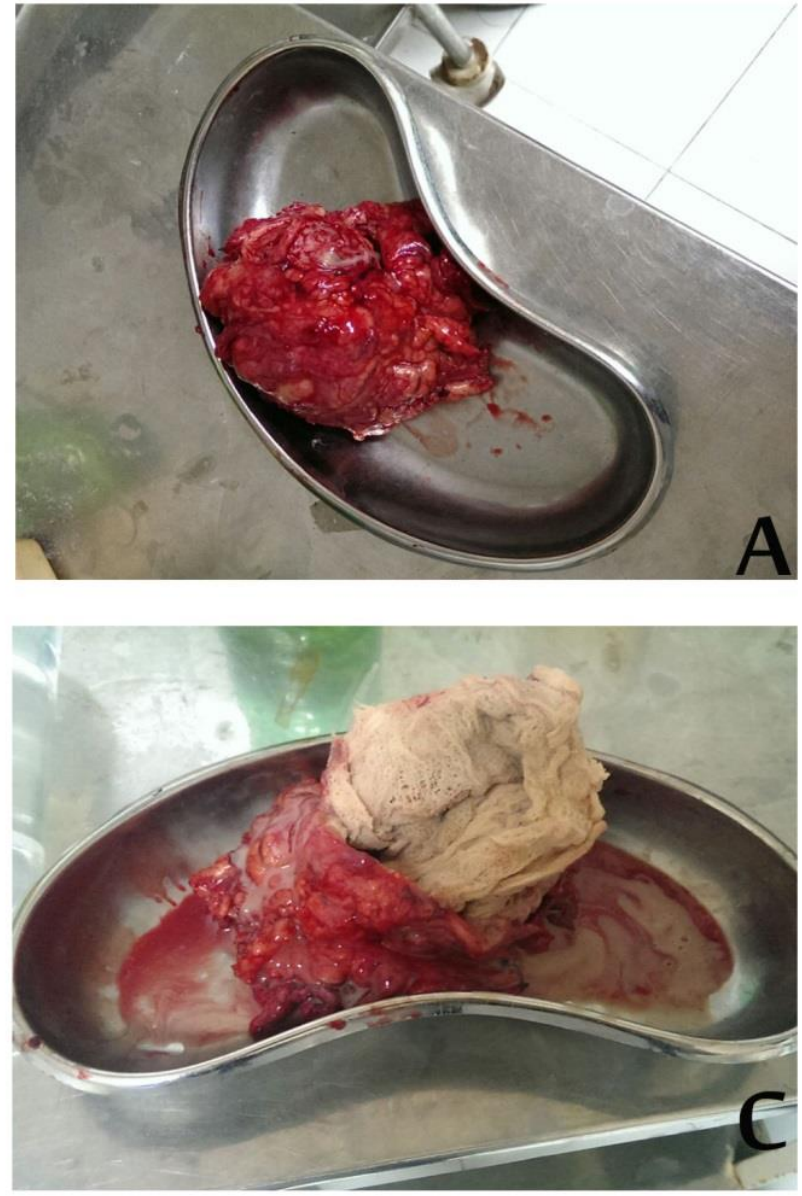

Sometimes the gauze which is entered into the lumen even migrates distally with peristaltic movements and eventually passes out of the rectum even without recognition [4].
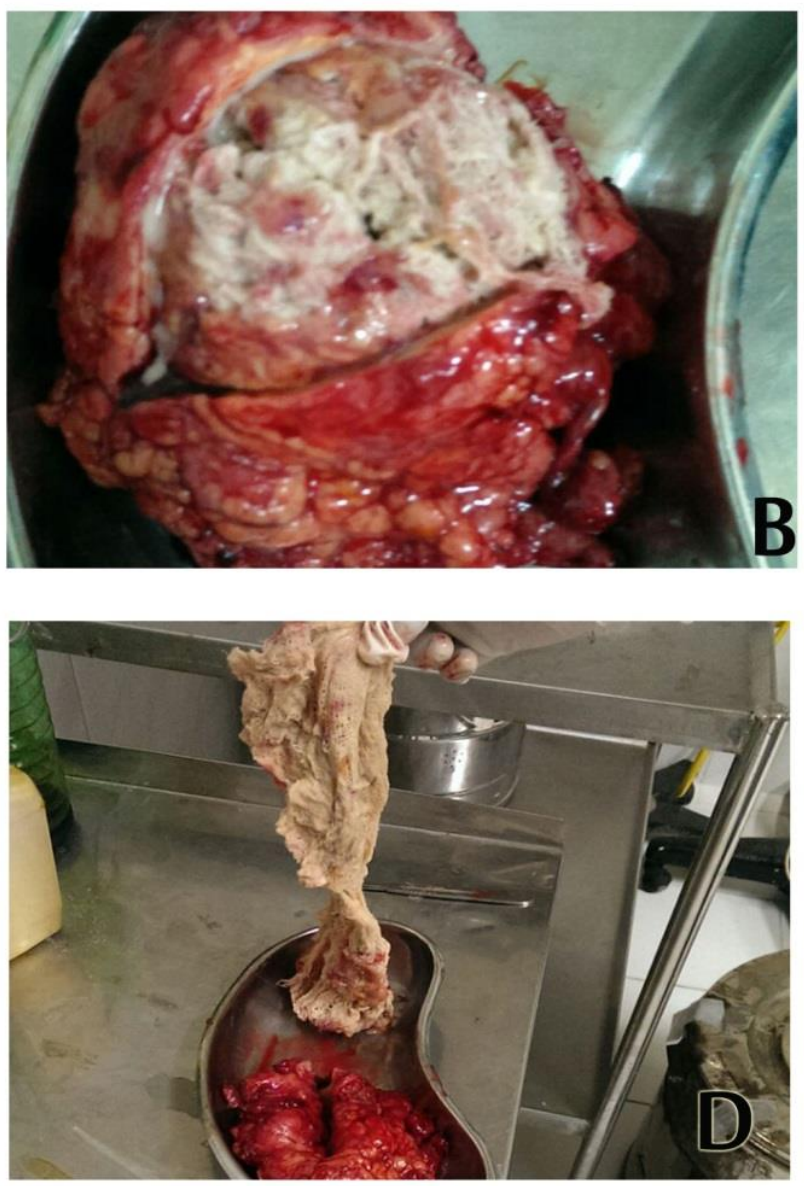

Fig. (1). A: Resected Specimen, B: Dissection of the mass, C: Gauze is seen coming out of the mass, D: Roll gauze after complete delivery.

Complete intra-luminal migration of RSS causing intestinal obstruction is a rarer entity and is difficult to diagnose due to vague presentation and inconclusive imaging. The symptoms are frequently labeled as non-specific and attributed to postop adhesions by many doctors in out-patient clinics and emergency rooms without proper investigation and management. So the patient remains undiagnosed for an extended period of time. Therefore, a high index of suspicion is necessary when a patient presents with non-specific abdominal complaints after abdominal/pelvic surgeries.

RSS may migrate transmurally into the lumen of any part of the gastro-intestinal tract including stomach [6], duodenum $[7,8]$, jejunum [9] ileum $[4,10]$ and colon $[11,12]$. It may cause an ulcer [8], perforation [11], fistula formation [3, 7], gastric outlet obstruction [7] and subacute/acute intestinal obstruction [2, 3, 4, 7] It may also remain silent/ asymptomatic after entering into the lumen and may even pass out through the rectum without causing much harm [5].

The removal of the gauze may require a re-laparotomy and re-exploration of the abdomen [1-6] but can also be removed endoscopically if it is present in the proximal part of the gastro-intestinal tract [6].

Many such cases are reported after emergency surgeries including emergency Cesarean Section and trauma laparotomies. The increased burden on emergency operating rooms, less availability of senior staff and senior surgeons at odd hours (like evening and night) and less trained junior surgeons doing such operations are some of the risk factors for retained or forgotten surgical gauzes.

Meticulous counting of surgical sponges and training of junior doctors and scrub nurses with emphasis on surgical 
swab counts is still the best way to reduce the incidence of retained swabs. A high index of suspicion is also necessary in post op patients who present with abdominal pain to prevent further damage to intra-abdominal organs and other life threatening consequence.

\section{AUTHORS CONTRIBUTION}

UA made substantial contribution to conception, design, preparation and editing of the manuscript.

AQA made contribution to design, literature search and manuscript writing.

SPA did review and final approval of the manuscript.

\section{ACKNOWLEDGEMENT}

This case study was represented by Dow university of Health Science.

\section{CONFLICT OF INTEREST}

Declared none.

\section{REFERENCES}

[1] Kundan KP, Shaifali KP, Kedar PG, Anuradha HP, Sahil SA, Raj PG. Intraluminal Migration of Surgical Sponge: Gossypiboma. Saudi J. Gastroenterol., 2010; 16 (3): 221-2.

[2] Chopra S, Suri V, Sikka P, Aggarwal N. A Case Series on Gossypiboma - Varied Clinical Presentations and Their Management. J. Clin. Disg. Res., 2015; 9(12).

[3] Margonis E, Vasdeki D, Diamantis A, Koukoulis G, Christodoulidis G, Tepetes K. Intestinal Obstruction and Ileocolic Fistula due to Intraluminal Migration of a Gossypiboma. Case Rep. Surg., 2016 18; 2016: 3258782.
[4] Grassi N, Cipolla C, Torcivia A, Bottino A, Fiorentino E, Ficano L, Pantuso G. Trans-visceral migration of retained surgical gauze as a cause of intestinal obstruction: a case report. J. Med. Case Reports. 2008; $2: 17$.

[5] Alis H, Soylu A, Dolay K, Kalayci M, Ciltas A. Surgical intervention may not always be required in gossypiboma with intraluminal migration world. J. Gastroenterol., 2007; 13(48):66057

[6] Sozutek A, Yormaz S, Kupeli H, Saban B. Transgastric migration of gossypiboma remedied with endoscopic removal: a case report. BMC Res Notes. 2013; 6: 413

[7] Manikyam SR1, Gupta V, Gupta R, Gupta NM. Retained surgical sponge presenting as a gastric outlet obstruction and duodeno-ileocolic fistula: report of a case. Surg. Today., 2002; 32(5): 426-8.

[8] Lv YX, Yu CC, Tung CF, Wu CC. Intractable duodenal ulcer caused by transmural migration of gossypiboma into the duodenum--a case report and literature review. BMC Surg. 2014; 14: 36 .

[9] Rafie BA, AbuHamdan OJ, Trengganu NS. Intraluminal migration of retained surgical sponge as a cause of intestinal obstruction. $J$. Surg. Case Rep., 2013; 5.

[10] Ali MA, Hussain A, Ghouse SM. Gossipyboma-a case report Indian J. Surg., 2012; 74(2): 177-8.

[11] Camera L, Sagnelli M, Guadagno P, Mainenti PP, Marra T, Scotto di Santolo M, Fei L, Salvatore M.Colonic perforation by a transmural and transvalvular migrated retained sponge: multidetector computed tomography findings. World J. Gastroenterol., 2014; 20(15): 4457-61.

[12] Cruz RJ Jr, Poli de Figueiredo LF, Guerra L. Intracolonic obstruction induced by a retained surgical sponge after trauma laparotomy. J. Trauma.2003; 55(5): 989-91. 